\title{
A Theory-Based Dietary Intervention for Overweight, Postpartum Mothers and Their Children Improves Maternal Vegetable Intake
}

\author{
Grace Falciglia'1, Julia Piazza1, Nicholas J. Ollberding2, Libbey Spiess ${ }^{3}$, Ardythe Morrow ${ }^{2}$ \\ ${ }^{1}$ Department of Nutritional Sciences, College of Allied Health Sciences, University of Cincinnati, Cincinnati, Ohio, USA \\ ${ }^{2}$ Division of Biostatistics and Epidemiology, Cincinnati Children's Hospital Medical Center, Cincinnati, Ohio, USA \\ ${ }^{3}$ Queen City Physicians-Pediatrics, Cincinnati, Ohio, USA \\ Email: grace.falciglia@gmail.com
}

How to cite this paper: Falciglia, G., Piazza, J., Ollberding, N.J., Spiess, L. and Morrow, A. (2017) A Theory-Based Dietary Intervention for Overweight, Postpartum Mothers and Their Children Improves Maternal Vegetable Intake. Open Journal of Obstetrics and Gynecology, 7, 679-692.

https://doi.org/10.4236/ojog.2017.77068

Received: February 24, 2017

Accepted: June 30, 2017

Published: July 3, 2017

Copyright ( 2017 by authors and Scientific Research Publishing Inc. This work is licensed under the Creative Commons Attribution International License (CC BY 4.0).

http://creativecommons.org/licenses/by/4.0/

\begin{abstract}
Objective: To evaluate the effectiveness of a dietary intervention to increase target vegetable intake in overweight, postpartum mothers; and their children. Methods: Overweight mothers attending their six-week postpartum follow-up visit and their infants ( $n=104$ pairs) were randomized to intervention or usual care groups during the time period 2008-2011. Mothers received four 60 minute education sessions with a nutrition professional and eight monthly follow-up phone calls. Counseling began at the obstetrician office and continued at the regularly scheduled pediatric visits. The primary study outcome was the change in maternal target vegetable intake. Secondary outcomes included child target vegetable intake and whether child vegetable intake was modified by exposure to breastfeeding. Mother/child energy intake and weight indices were also assessed. Outcomes were measured at baseline (6-weeks postpartum), 6, 12 (post-intervention), and 18 (follow-up) months. Mixed-effects models were used to estimate the impact of the dietary intervention on study outcomes relative to usual care. Results: Mothers randomized to the intervention had greater consumption of target vegetables at 6 , 12 and 18 months $(P<0.01, P<0.01$ and $P=0.03$, respectively). There were no differences between groups in maternal energy intake, body mass index, or child target vegetable or energy intake. The child's target vegetable intake at 12 months was related to the mother's intake at 6 months $(P=0.03)$, however, this relationship was not modified by exposure to breastfeeding. Conclusion: A dietary intervention targeting the diet of the mother/child dyad resulted in improved maternal vegetable intake.
\end{abstract}

\section{Keywords}

Prevention of Childhood Obesity, Overweight Postpartum Women, Maternal 
Diets, Infant and Toddler Diets, Dietary Intervention

\section{Introduction}

Increased consumption of vegetables is a key component of dietary recommendations aimed at improving health and preventing disease [1]. However, fewer than $15 \%$ of adults and children report meeting the recommendations for total vegetables; with fewer consuming the recommended number of servings of nutrient dense dark-green and deep-yellow vegetables. Previous research has reported that transition to motherhood is associated with a decrease in vegetable intake [2]. It is critical for postpartum women to develop healthy eating patterns since many women enter pregnancy overweight and gain excess weight during pregnancy [3]. In addition, growing evidence suggests that overweight mothers are more likely to have overweight children [4].

It has been shown that not only children in general struggle with high weight but the youngest children (infants and toddlers) are now experiencing similar problems [5]. Therefore, it is important to encourage the establishment of healthy dietary patterns as early as possible [6]. The national Feeding Infants and Toddlers Study (FITS) surveyed young children's diets and found them to be high in calories, fat, and sugar and markedly low in vegetables [7]. Consumption of nutrient dense vegetables is critical for energy balance because they are low in calories, high in fiber and water [8]. Then, a greater volume of foods low in calories can be eaten compared to foods with high energy value, leading to satiety with fewer calories [9].

Expecting children to accept vegetables is a challenge due to their innate dislike for bitter flavors and their predisposition towards neophobia, unwillingness to try novel foods [10]. The first year of life provides a unique window of opportunity to introduce children to vegetables, for most infants around 6 months of age, since the neophobic response is less developed at this time; fewer exposures to a new food are needed to overcome neophobia [11]. Additionally, if the child is breastfed, he or she is exposed to flavors of foods including vegetables from the mother's diet through the breast milk [12] [13] [14]. Because the mother, among others, affects the child's diet directly by choosing what the child is offered to eat, and indirectly by flavors transmitted through breast milk, improving the diets of infants and children may be dependent on improving the maternal diet. To date, there have been no studies showing that modifying the maternal diet can affect the child's diet in a real-life setting. Therefore, there is a need to develop an effective dietary intervention that takes into account the complex interplay between the diets of mother/child dyad.

The overall goal of this study was to promote the adoption of healthy eating patterns in overweight mothers and their children using the Social Cognitive Theory (SCT) as the conceptual framework. Specifically, this study was designed to evaluate the effectiveness of a year-long dietary intervention on increasing the 
intake of deep-yellow and dark-green vegetables in overweight mothers when compared to women receiving usual care. Secondary outcomes of interest included whether the dietary intervention would result in improvements in maternal energy intake and body mass index (BMI), as well as improvements in child target vegetable intake, energy intake, and weight-for-length $\mathrm{z}$-score (WLZ). Accordingly, we had two hypotheses: 1) Mother/child dyads receiving the dietary intervention would be more likely to improve their diets through a greater increase in intake of target vegetables leading to a greater decrease in caloric intake and normalization of body weight-for-height/length when compared to mother/child dyads receiving usual care, and 2) The child's vegetable intake would be associated with the mother's vegetable intake modified by degree of exposure to breastfeeding.

\section{Materials and Methods}

\subsection{Study Design and Participants}

This was a randomized controlled dietary intervention with integration of services among obstetricians, pediatricians and nutrition professionals. Women attending their 6-week postpartum follow-up visit at a large obstetrics clinic in the Cincinnati metropolitan area were screened for inclusion between March 2008 and April 2011. Inclusion criteria consisted of: BMI $\geq 25$ with no co-morbidities; 21 - 35 years of age; given birth to a singleton, full-term infant; a target vegetable intake (deep-yellow and dark-green vegetables) less than or equal to 1.5 servings/day (based on current vegetable intake from pilot data) [15]; full medical clearance from a physician to participate; no special diet prescribed; limited use of alcohol (one alcoholic drink/day); and no use of illegal drugs or tobacco. Mothers that did not meet those criteria were excluded for participation. Written informed consent was obtained from all eligible participants. Study procedures were approved by the Institutional Review Board of the University of Cincinnati.

Based on our preceding pilot study [15], we calculated a total of 120 mothers, with even allocation to treatment and control conditions, were required to provide $80 \%$ power to detect a difference in total target vegetable intake of 0.55 servings/day between the intervention and usual care groups at one year (twotailed alpha $=0.05)$. Out of the 914 participants assessed, 263 met the eligibility criteria (Figure 1). Of those, 104 consented to participate in the study and were scheduled for baseline assessment followed by randomization to either dietary intervention $(n=52)$ or usual care $(n=52)$. Participants received a $\$ 25$ food gift certificate for completing each assessment.

\subsection{Conditions}

Intervention Group. Dietary counseling was provided by nutrition professionals with graduate level training in nutrition and theory-based nutrition education. Counseling began at the obstetrician office and continued at the regularly scheduled pediatric visits. The dietary intervention was tailored to the socio de- 


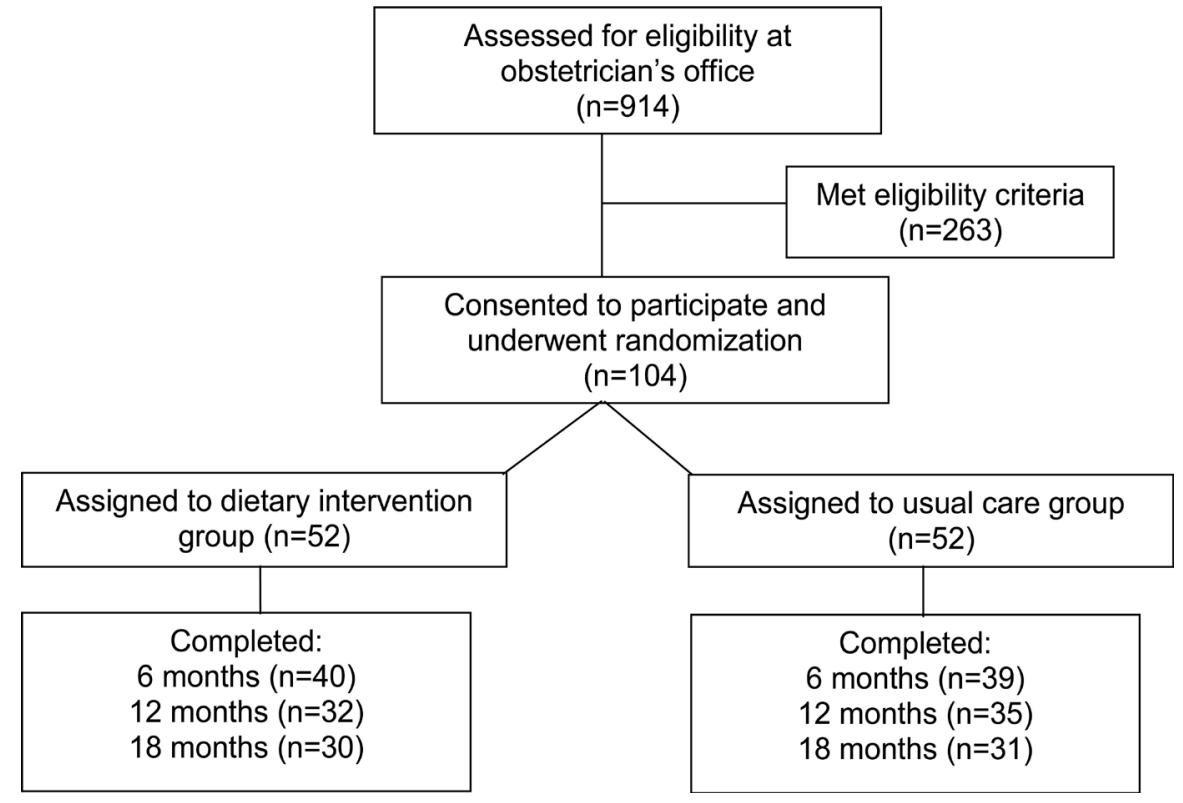

Figure 1. Flow chart of study recruitment and retention.

mographic characteristics of the participants as learned from our preceding pilot study [15]. The intervention focused on consuming a healthy diet, with an emphasis on deep-yellow and dark-green vegetables. Those vegetables are underrepresented in the majority of Americans' diets and can be eaten from infancy through adulthood. Target vegetables included carrots, winter squash, sweet potatoes, broccoli, spinach, and peas. The Social Cognitive Theory used in this intervention explains behavior in terms of a reciprocal model in which behavior, personal factors, and environmental influences interact [16]. The main theory constructs (mediators of change) incorporated into this intervention were selfefficacy (confidence in choosing vegetables in a variety of settings and circumstances), along with self-regulation of behavior (goal setting, action planning, self-monitoring, and problem solving). The manual developed for the individualized nutrition education sessions included three modules: Maternal diet (calorie level appropriate for gradual weight loss while supporting lactation), Intro to solid foods and Transition into toddler foods. All modules included strategies to gain skills in the selected constructs, such as tips on choosing and preparing deep-yellow and dark-green vegetables, as well as instruments to track improvements in behavioral self-regulation. Monthly phone calls were directed to discuss the progress of both the mother and child, set new goals, and problem solve. Mothers were encouraged to make gradual dietary changes to comply with the US Department of Agriculture (USDA) food guide MyPyramid [17] and achieve the intervention goal of consuming at least 2 servings of the target vegetables and a total of 5 servings of vegetables daily. Mothers were also advised to follow the Start Healthy Feeding Guidelines for infants and toddlers [18]. Special emphasis was placed on the same target vegetables that were promoted in the maternal diet. At the time of introducing complementary foods, the vegetable intake goal was to eat 3 servings of vegetables a day ( 1 serving $=1$ tablespoon), 
with at least one serving being a target vegetable. The goal for children transitioning to toddler foods was to eat at least 3 servings of vegetables each day ( 1 serving $=1 / 4$ cup [about 4 tablespoons]), with at least one serving being a target vegetable. Table 1 provides details of the intervention format, content, and food plans based on type of feeding. The nutrition professionals provided all faceto-face dietary counseling and made all telephone contacts with study participants. For each participant, the telephone contact person remained constant throughout the intervention. An intervention curriculum and call scripts were developed to provide consistency in information delivery.

Usual Care Group. At baseline, standard educational materials were mailed to the mothers on healthy eating according to the USDA food guide MyPyramid recommended for breastfeeding or formula feeding needs [17]. A handout with basic information on starting solid foods and transitioning to toddler foods was also included [18]. Formal dietary counseling sessions or phone calls were not included in the usual care group.

\subsection{Measures}

Dietary Intake. All measures were evaluated at baseline (6-weeks postpartum), 6,12 , and 18 (follow-up) months. Dietary intake for the mother/child dyads was assessed via 3 unannounced, interviewer-administered 24-hour dietary recalls conducted over a one-week time span at each evaluation point. The recalls were

Table 1. Intervention format, content, and food plan.

\begin{tabular}{|c|c|c|}
\hline Format & \multicolumn{2}{|c|}{$\begin{array}{l}\text { One-year intervention consisting of four } 60 \text { minute face-to-face } \\
\text { education sessions with nutrition educator }{ }^{\mathrm{a}} \text { and participant. Eight } \\
\text { monthly follow-up phone calls ( } 15 \text { - } 30 \text { minutes each) by same } \\
\text { nutrition educator. }\end{array}$} \\
\hline Content & \multicolumn{2}{|c|}{$\begin{array}{l}\text { Session } 1 \text { ( } 2 \text { months post-delivery) manual with three modules } \\
\text { provided to participant and first module explained: Maternal diet; } \\
\text { Sessions } 2 \text { and } 3 \text { ( } 4 \text { and } 6 \text { months post-delivery) second module } \\
\text { explained: Intro to solid foods; Session } 4 \text { ( } 9 \text { months post-delivery) } \\
\text { third module explained: Transition into toddler foods. All modules } \\
\text { included food monitoring tools, guidelines for goal-setting, action } \\
\text { planning, self-rewarding and problem solving. Monthly phone calls } \\
\text { discussed mother and child progress. }\end{array}$} \\
\hline Mother Food plan & Breast Feeding & Formula-Feeding \\
\hline Calories $^{\mathrm{b}}$ & 2000 & 1500 \\
\hline \multicolumn{3}{|c|}{ Servings/day (serving size) } \\
\hline Grains & $6(10 z)$ & $5(1 \mathrm{oz})$ \\
\hline Vegetables & 5 (1/2 cup) & 5 (1/2 cup) \\
\hline Fruit & 4 ( $1 / 2$ cup $)$ & 2 (1/2 cup) \\
\hline Milk & 3 (1 cup) & 3 (1 cup) \\
\hline Meat and Beans & $2(3 \mathrm{oz}$ cooked $)$ & $1-2(3 \mathrm{oz}$ cooked $)$ \\
\hline
\end{tabular}

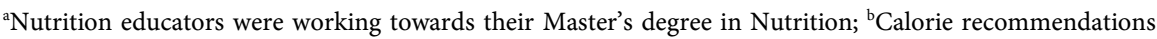
for breastfeeding mothers were determined as follows: 1700 calories/d (daily intake for non-breast feeding mothers, according to the 1994-96 USDA Continuing Survey of Food Intake by Individuals), +500 extra calories/d (to support breast feeding), -200 calories/d (to see 2 pounds per month weight loss). Calorie recommendations for formula feeding mothers were determined as follows: 1700 calories/d (daily intake for non-breast feeding mothers), -200 calories/d (to see 2 pounds per month weight loss). 
completed over the phone by trained interviewers from the Cincinnati Center for Nutritional Research and Analysis at the Cincinnati Children's Hospital Medical Center using the Nutrition Data System for Research (NDS-R: 2009, 2010, 2011, University of Minnesota, MN). The interview method employs multiple passes to enhance detail and limit omissions. To assist in portion size estimation mothers were provided with a 2-dimensional measuring guide [19]. Target vegetables contained in mixed dishes were included in the vegetable serving counts (NDRS codes: 0100 for dark-green vegetables and 0200 for deepyellow vegetables). Mothers were also asked specific questions regarding intensity and duration of breastfeeding and the age at which their infants were first fed infant formula or food. Dietary recalls for the mothers were analyzed for daily average energy intake (kcal) and food groups (number of servings) according to the 2010 Dietary Guidelines for Americans [1]. Classification of foods for the child followed the food grouping developed for the 2008 Feeding Infants and Toddlers Study [20].

Anthropometrics. The maternal height and weight measurements and child weight and length measurements were collected at each evaluation time by trained staff members using standardized procedures. Measures were taken twice and the average of the two readings was retained. Maternal height and weight were measured wearing light clothing, without shoes, using a digital portable scale, with stadiometer (Model 869, Seca, Chino, CA). Child weight and recumbent length was taken while the child was unclothed, using a digital baby scale, with stadiometer (Model DS4100, Batavia, IL). Children's z-scores were calculated as weight-for-length $z$-score (WLZ) using the Centers for Disease Control and Prevention growth curves [21].

Breastfeeding Index (BFI). The duration and intensity of breastfeeding was measured using the Cincinnati Breastfeeding Cohort Baseline Questionnaire [22]. The metric for breastfeeding exposure was a cumulative BFI of duration times intensity of the infant's exposure to breast milk over the 6 month period $(0$ $-6,7-12,13-18)$ preceding each evaluation time. Specifically, duration was calculated in months and converted to weeks based on the age of the child. Intensity was calculated as a ratio of the number of human milk feedings divided by the total number of feedings (formula, water, other foods) the day before each assessment was conducted (all breast milk = ratio 1; breast milk plus formula/other foods $=0.1-0.9$; all formula/other foods $=$ ratio 0 ).

\subsection{Statistical Analysis}

Baseline comparisons of mother and child demographic characteristics were conducted using two-sample t-tests for continuous variables and $\chi^{2}$ tests for categorical variables. All analyses assessing mother/child dietary and anthropometric outcomes in response to the dietary intervention were conducted in accordance with the intent-to-treat (ITT) principle. Mixed-effects models were used to calculate the impact of the dietary intervention study outcomes relative to usual care. The model was chosen a priori and included treatment assignment, 
time, baseline response, and a time by treatment interaction term as fixed effects and subject as a random effect.

The primary study outcome was the change in maternal daily total target vegetable intake (servings/d) at one year. Secondary outcomes included the maternal energy intake and BMI and child total target vegetable intake, energy intake, and WLZ. Post hoc sensitivity analyses were also conducted to assess the robustness of the primary study outcome to incomplete randomization of baseline maternal energy intake and attrition. Maternal baseline energy intake was added as a fixed effect to the primary analysis model to assess the potential impact of incomplete randomization at baseline. To assess whether attrition may have biased the results, factors predictive of withdraw were identified using logistic regression. These predictors were then included in multiple imputation (MI) models ( $n=20$ datasets) as auxiliary variables. As a final sensitivity analysis the missing data were assumed to be missing not at random (MNAR) and a pattern-mixture model was used to impute values for those who withdrew by using the observed distribution of target vegetable intake in the usual care group at that time point. This model assumes that participants in the dietary intervention group who drop out reap no further benefits from the intervention and their distribution of target vegetable intake resembles that of those receiving usual care.

In additional analyses, the child's target vegetable intake at 12 months was regressed on the mother's target vegetable intake at 6 months to assess the association between mother and child target vegetable intake. An interaction term for mother's target vegetable intake and BFI at 6 months was included to assess whether the association was modified by exposure to breastfeeding. Self-efficacy in choosing vegetables in a variety of settings and circumstances was posited as a primary mediator through which the intervention would influence maternal target vegetable intake; however, it was not examined in this analysis since it was found to be high among all mothers at baseline and to remain stable over the study period. Data on self-regulation was not collected. All analyses used SAS (version 9.3, 2011, SAS Institute Inc.) and $p$-values $\leq 0.05$ were considered to indicate statistical significance.

\section{Results}

Participant demographic characteristics according to study group are shown in Table 2. The mean mother and child age at baseline was $29 \mathrm{y}$ and 1.9 months, respectively. Approximately half of the infants were male. Similar proportions of mothers randomized to the dietary intervention (60\%) and usual care $(67 \%)$ reported initiation of breastfeeding by 6 weeks. Over two-thirds of the mothers enrolled in the study were non-Hispanic white, reported educational attainment of a college degree or beyond, were employed, and were married. The mean BMI of mothers at baseline was $30 \mathrm{~kg} / \mathrm{m}^{2}$ and the mean WLZ of children was approximately -0.4 units as shown in Table 3. Participants in the dietary intervention and usual care groups did not differ on any measured demographic charac- 
Table 2. Baseline characteristics of mothers and children according to study group.

\begin{tabular}{|c|c|c|c|}
\hline Characteristic & Intervention $(n=52)$ & Usual Care $(n=52)$ & $P$-value ${ }^{\mathrm{a}}$ \\
\hline Maternal age (y), mean (SD) & $28.8(4.8)$ & $29.3(4.0)$ & 0.52 \\
\hline Infant age (months), mean (SD) & $1.9(0.4)$ & $1.9(0.5)$ & 0.74 \\
\hline Infant sex male, $n(\%)$ & $25(48.1)$ & $27(51.9)$ & 0.70 \\
\hline First born, $n(\%)$ & $26(50.0)$ & $23(44.2)$ & 0.56 \\
\hline Ever breastfed, $n(\%)$ & $31(59.6)$ & $35(67.3)$ & 0.42 \\
\hline \multicolumn{4}{|l|}{ Maternal education, $n(\%)$} \\
\hline Some college or less & $17(32.7)$ & $18(34.6)$ & \\
\hline College graduate or beyond & $35(67.3)$ & $34(65.4)$ & 0.84 \\
\hline Mother employed, $n(\%)$ & $35(67.3)$ & $37(71.2)$ & 0.67 \\
\hline Mother married, $n(\%)$ & $37(71.2)$ & $40(76.9)$ & 0.50 \\
\hline \multicolumn{4}{|l|}{ Maternal race, $n(\%)$} \\
\hline Non-Hispanic White & $39(75.0)$ & $38(73.1)$ & \\
\hline Other & $13(25.0)$ & $14(26.9)$ & 0.82 \\
\hline
\end{tabular}

${ }^{a} P$-values for two-sample $t$ tests for continuous variables and $\chi^{2}$ tests for categorical variables testing differences between intervention and usual care groups.

Table 3. Differences in dietary and anthropometric outcomes between intervention and usual care groups.

\begin{tabular}{|c|c|c|c|c|c|c|c|c|c|c|c|}
\hline & & $\begin{array}{l}\text { Mean (SD) } \\
\text { Baseline }\end{array}$ & $\begin{array}{l}\text { Mean (SD) } \\
\quad 6 \text { mo. }\end{array}$ & $\begin{array}{l}\text { Mean (SD) } \\
12 \text { mo. }\end{array}$ & $\begin{array}{l}\text { Mean (SD) } \\
18 \text { mo. }\end{array}$ & $\begin{array}{c}\Delta_{\text {Intervention }}- \\
\Delta_{\text {Usual }} \\
\text { Mean (SE) } \\
6 \text { mo. }^{\text {a }}\end{array}$ & $\begin{array}{c}\Delta_{\text {Intervention }}- \\
\Delta_{\text {Usual }} \\
\text { Mean (SE) } \\
12 \text { mo. }^{\text {a }}\end{array}$ & $\begin{array}{c}\Delta_{\text {Intervention }}- \\
\Delta_{\text {Usual }} \\
\text { Mean (SE) } \\
18 \text { mo. }^{\mathrm{a}}\end{array}$ & $\begin{array}{l}P \text {-value }{ }^{\mathrm{b}} \\
6 \mathrm{mo} .\end{array}$ & $\begin{array}{l}P \text {-value } \\
12 \mathrm{mo}\end{array}$ & $\begin{array}{l}P \text {-value } \\
18 \text { mo. }\end{array}$ \\
\hline \multicolumn{12}{|c|}{ Primary Outcomes: Maternal } \\
\hline \multirow{2}{*}{$\begin{array}{l}\text { Total target } \\
\text { vegetables } \\
\text { (servings/d) }\end{array}$} & Intervention & $0.7(0.9)$ & $1.3(1.2)$ & $1.1(0.8)$ & $0.8(0.8)$ & $0.8(0.2)$ & $0.6(0.2)$ & $0.4(0.2)$ & $<0.01$ & $<0.01$ & 0.03 \\
\hline & Usual Care & $0.4(0.5)$ & $0.5(0.5)$ & $0.4(0.7)$ & $0.4(0.4)$ & & & & & & \\
\hline \multicolumn{12}{|c|}{ Secondary Outcomes: Maternal } \\
\hline \multirow[t]{2}{*}{ Energy $(\mathrm{kcal} / \mathrm{d})^{\mathrm{c}}$} & Intervention & $1900.0(487.6)$ & $1648.0(378.4)$ & $1549.7(336.9)$ & $1544.9(362.3)$ & -106.9 & -148.3 & $-90.5(95.1)$ & 0.19 & 0.11 & 0.35 \\
\hline & Usual Care & $1658.5(538.0)$ & $1634.8(494.9)$ & $1576.1(533.4)$ & $1519.2(419.4)$ & $(01.0)$ & (32.1) & & & & \\
\hline \multirow[t]{2}{*}{$\begin{array}{l}\text { Body mass index } \\
\qquad\left(\mathrm{kg} / \mathrm{m}^{2}\right)\end{array}$} & Intervention & $31.3(4.7)$ & $30.1(4.9)$ & $29.7(4.7)$ & $30.2(4.8)$ & $0.0(0.4)$ & $0.5(0.5)$ & $0.4(0.6)$ & 0.91 & 0.35 & 0.53 \\
\hline & Usual Care & $29.7(4.5)$ & $28.2(4.0)$ & $27.4(4.3)$ & $28.0(4.5)$ & & & & & & \\
\hline \multicolumn{12}{|c|}{ Secondary Outcomes: Infants/Toddlers } \\
\hline \multirow{2}{*}{$\begin{array}{l}\text { Total target } \\
\text { vegetables } \\
\text { (grams/d) }\end{array}$} & Intervention & $0.0(0.0)$ & $35.8(40.4)$ & $46.6(51.2)$ & $31.6(41.8)$ & $-2.1(9.5)$ & $5.4(12.1)$ & $5.4(9.6)$ & 0.83 & 0.66 & 0.57 \\
\hline & Usual Care & $0.0(0.0)$ & $37.9(45.3)$ & $42.1(49.5)$ & $26.6(34.6)$ & & & & & & \\
\hline \multirow[t]{2}{*}{ Energy (kcal/d) } & Intervention & $551.7(105.1)$ & $643.5(139.4)$ & $948.2(249.1)$ & $1081.2(230.8)$ & $9.1(29.6)$ & $32.4(56.4)$ & $-33.2(60.1)$ & 0.76 & 0.57 & 0.58 \\
\hline & Usual Care & $545.1(127.7)$ & $628.1(133.3)$ & $906.7(220.4)$ & $1091.5(239.3)$ & & & & & & \\
\hline $\begin{array}{l}\text { Weight-for-length } \\
\text { z-score (SD units) }\end{array}$ & Intervention & $-0.7(1.9)$ & $-0.6(1.4)$ & $-0.2(1.1)$ & $-0.4(1.6)$ & $-0.6(0.3)$ & $-0.4(0.3)$ & $-0.7(0.3)$ & 0.03 & 0.10 & 0.05 \\
\hline
\end{tabular}

Abbreviations: SD, standard deviation, SE, standard error; Notes: Maximum likelihood-based estimates for the intent-to-treat analysis obtained from linear mixed-effects models; ${ }^{\mathrm{a}}$ Values are the mean difference (SE) between intervention and usual care groups; ${ }^{\mathrm{b}} P$-value for the difference between intervention and usual care groups; ${ }^{\mathrm{c}}$ Maternal energy intake differed between the intervention and usual care group at baseline $(P=0.02)$. 
teristic or study measure at baseline other than maternal energy intake. The mean baseline energy intake was $1900( \pm 488) \mathrm{kcal} / \mathrm{d}$ for mothers in the dietary intervention group and $1659( \pm 538)$ for mothers receiving usual care $(P=0.02)$. The retention of mother/child dyads over the follow-up was as follows: 6 months postpartum (intervention 77\%, usual care 75\%); 12 months postpartum (intervention $62 \%$, usual care $67 \%$ ); and 18 months postpartum (intervention $58 \%$, usual care 60\%). Age, ever breastfeeding, higher education, being married, and non-Hispanic white race were associated with withdrawal.

Baseline mean total target vegetable intake was similar for mothers randomized to the dietary intervention $(0.7 \pm 0.9$ servings/d) or usual care $(0.4 \pm 0.5$ servings/d). Compared to mothers receiving usual care, mothers in the dietary intervention group increased their consumption of target vegetables by 0.8 $( \pm 0.2)$ servings/d at 6 months, $0.6( \pm 0.2)$ servings/d at 12 months, and $0.4( \pm 0.2)$ servings/d at 18 months (6 month follow-up). Differences between groups in the change in target vegetable consumption from baseline were statistically significant at all-time points $(p \leq 0.03)$. The estimated ITT effect for the difference between groups was generally similar in sensitivity analyses examining the robustness of the study findings to incomplete randomization and attrition (Supplemental Table 1). In analyses adjusting for the maternal baseline energy intake the estimated treatment effect was $0.8( \pm 0.2)$ servings/d at 6 months $(P<0.01)$, $0.6( \pm 0.2)$ servings/d at 12 months $(P<0.01)$, and $0.3( \pm 0.2)$ servings/d at 18 months $(P=0.04)$. Age, ever breastfeeding, education, marital status, and race were associated with withdrawal at one or more time points and included the MI model as auxiliary variables. Estimated mean differences between groups with respect to the change from baseline for this model were $0.7( \pm 0.2)$ servings/d at 6 months $(P<0.01), 0.8( \pm 0.2)$ servings/d at 12 months $(P<0.01)$, and $0.4( \pm 0.2)$ servings/d at 18 months $(P=0.06)$. For the pattern-mixture model assuming that the data were MNAR and dietary intake for drop outs in the dietary intervention was similar to those receiving usual care, estimated mean differences between groups were $0.5( \pm 0.2)$ servings/d at 6 months $(P<0.01), 0.4( \pm 0.2)$ servings/d at 12 months $(P=0.02)$, and $0.3( \pm 0.1)$ servings/d at 18 months $(P=$ $0.03)$; highlighting the robustness of the main findings to different assumptions for the missing data mechanism.

Secondary outcomes including maternal energy intake and BMI, as well as child total target vegetable or energy intake did not differ between the intervention and usual care groups $(p>0.10)$ (Table 3 ). Compared to mothers receiving usual care, children of intervention mothers had a difference in the change from baseline of $-0.6( \pm 0.3)$ in WLZ at six months $(P=0.03)$. In addition, in linear regression analyses including intervention and usual care mothers, the maternal target vegetable intake at 6 months was associated with the child target vegetable intake at 12 months. Within the range of the data, for every additional $1 / 2$ serving/d of target vegetables consumed by the mother at 6 months the child consumed an additional 7.2 grams/d of target vegetables $(\beta=7.23, \mathrm{SE}=3.67, P=$ 0.05 ; data not shown) at 12 months. This relationship was not modified by de- 
gree of exposure to breastfeeding.

\section{Discussion}

This study provides evidence for the effectiveness of a tailored behavioral intervention to improve the diet of overweight, postpartum mothers. Mothers in the intervention group showed greater improvements in target vegetable consumption relative to the usual care group at 6 and 12 months. In addition, the improvement in vegetable consumption persisted up to 18 months, 6 months after the completion of the intervention. This increase in vegetable intake is consistent with the results from the preceding pilot study [15] and other previous studies. For example, a comprehensive review of the literature on the efficacy of dietary interventions to modify vegetable and fruit intakes showed that 17 of 22 studies reported an average increase of 0.6 servings/day [23]. A more recent systematic review of behavioral interventions reported an average increase in the consumption of vegetables and fruits of +1.13 servings/d in adults [24]. This finding is important, as it shows that a tailored behavioral intervention can have a sustained impact on increasing the consumption of deep-yellow and dark-green vegetables, which are under consumed in the US diet, and overweight, postpartum women.

The dietary data for children show that the intervention and usual care groups met and surpassed the goal of eating 1 serving ( $\sim$ tablespoon; $\sim 15 \mathrm{~g}$ ) of target vegetables a day at 6 months. This finding was expected as this is the time when infants are introduced to solid foods including yellow and green vegetables. At 12 and 18 months when vegetables start to disappear from the children's diet [7], neither group met the goal of 4 tablespoons/day; however the intervention group's intake of target vegetable remained different in a positive way, although no significant, as compared to the control group. A small difference in daily servings may have clinical significance given that preference for vegetables increases with exposure and preference is a strong determinant of food consumption [10].

Compared to mothers receiving usual care, infants/toddlers of intervention mothers had a smaller increase in WLZ at 6 months; however, the lack of a statically significant effect on child target vegetable and total energy intake limits the interpretation of this finding. Studies of longer duration and designed to detect difference in WLZ or adiposity in later childhood would be required to determine whether the dietary intervention provides a sustained benefit with respect to preventing pediatric overweight and obesity.

One notable finding is that the mother's target vegetable intake at 6 months was related to the child's target vegetable intake at 12 months. This period represents an important time of transitioning to table foods and characterized by a decrease in total vegetable intake [7]. Previous studies had suggested that vegetable flavors in breast milk may increase children vegetable acceptance [12] [13] [14]. In the present study, the association between the child's and mother's target vegetable intake was not modified by exposure to breastfeeding; however, our ability to detect such an effect was limited. In addition, compared to pre- 
vious studies, we used current dietary guidelines to determine the number of daily servings and portion size for target vegetables [18] and emphasized 6 target vegetables which may be too small of an amount and/or too many vegetables at one time for breastfeeding to influence food consumption. Larger studies specifically designed to examine these interactions are needed.

Strengths of this study included providing nutrition education to mother/child dyads through a combination of individual counseling sessions, coinciding with visits to the obstetrician and pediatrician, and follow up phone calls. Also, while information on healthy diet is a necessary component of any dietary intervention, this particular nutrition education program was theory-based and conducted by trained nutrition professionals who taught parents' skills and strategies to manage behavior and implement change. Notably, self-efficacy and self-regulation skills seemed particularly beneficial to the mothers, who like most postpartum women are ready for action and or change [16]. Furthermore, for the mothers receiving nutrition intervention, improvements in target vegetable consumption occurred at 6 and 12 months and were maintained for 6 months after the completion of the intervention.

There are also limitations. Due to the rigorous nature of this intervention, there was appreciable attrition over an 18-month period. However, the increase in target vegetable intake in intervention mothers was robust to sensitivity analyses accounting for factors predictive of withdrawal and assuming no treatment effect for those lost to follow-up. In addition, the study sample was not representative of all overweight, postpartum women. The study included predominantly white, highly educated women; therefore additional data are needed from various socioeconomic levels and ethnic backgrounds. We also cannot rule out that receipt of the dietary intervention did not result in post-randomization differences in the ability to accurately self-report dietary intake, as no objective measure of energy or target vegetable intake was available. Lastly, the study was not powered to detect differences in secondary outcomes.

\section{Conclusion}

In conclusion, the results of this study in overweight, postpartum women show that a tailored, theory-based behavioral intervention, targeting the diet of the mother and child dyad, can increase maternal consumption of under consumed deep-yellow and dark-green vegetables. The integration of nutrition education within regular visits to the obstetrician and pediatrician seems to be an effective strategy in developing healthy food behaviors in overweight, postpartum women.

\section{Funding}

This study was supported by a research grant from the National Institute of Child Development (1R15HD055598-01).

\section{References}

[1] US Department of Agriculture and US Department of Health and Human Services 
(2010) Nutrition and Your Health: Dietary Guidelines for Americans. 7th Edition, U.S. Government Printing Office, Washington, DC.

[2] Olson, C.M. (2005) Tracking of Food Choices across the Transition to Motherhood. Journal of Nutrition Education and Behavior, 37, 129-136. https://doi.org/10.1016/S1499-4046(06)60267-4

[3] Esposito, L., Fisher, J.O., Mennella, J.A., Hoelscher, D.M. and Huang, T.T. (2009) Developmental Perspectives on Nutrition and Obesity from Gestation to Adolescence. Prevention of Chronic Diseases, 6, 1-11.

[4] Oken, E. and Gillman M.W. (2003) Fetal Origins of Obesity. Obesity Research, 11, 496-506. https://doi.org/10.1038/oby.2003.69

[5] Ogden, C.L., Carroll, M.D., Curtin, L.R., Lamb, M. and Flegal, K.M. (2010) Prevalence of High Body Mass Index in US Children and Adolescents, 2007-2008. Journal of the American Medical Association, 303, 242-249. https://doi.org/10.1001/jama.2009.2012

[6] Huang, T.T. and Glass T. (2008) Transforming Research Strategies for Understanding and Preventing Obesity. Journal of the American Medical Association, 300, 1811-1813. https://doi.org/10.1001/jama.300.15.1811

[7] Siega-Riz A.M., Deming, D.M., Reidy, K.C., Fox, M.K., Condon, E. and Briefel R.R. (2010) Food Consumption Patterns of Infants and Toddlers: Where Are We Now? Journal of the American Dietetic Association, 110, S38-S51. https://doi.org/10.1016/j.jada.2010.09.001

[8] Rolls, B.J., Ello-Martin, J.A. and Tohill B.C. (2004) What Can Intervention Studies Tell Us about the Relationship between Fruit and Vegetable Consumption and Weight Management? Nutrition Reviews, 62, 1-17. https://doi.org/10.1111/j.1753-4887.2004.tb00001.x

[9] Epstein, L.H., Gordy, C.C., Raynor, H.A., Beddome, M., Kilanowski, C.K. and Paluch, R. (2001) Increasing Fruit and Vegetable Intake and Decreasing Fat and Sugar Intake in Families at Risk for Childhood Obesity. Obesity Research, 9, 171-178. https://doi.org/10.1038/oby.2001.18

[10] Birch, L.L. (1999) Development of Food Preferences. Annual Review of Nutrition, 19, 41-62. https://doi.org/10.1146/annurev.nutr.19.1.41

[11] Falciglia, G.A., Couch, S.C., Siem-Gribble, L., Pabst, S.M. and Frank, R. (2000) Food Neophobia in Childhood Affects Dietary Variety. Journal of the American Dietetic Association, 100, 1474-1478. https://doi.org/10.1016/S0002-8223(00)00412-0

[12] Mennella, J.A., Jagnow, C.L. and Beauchamp, G.K. (2001) Prenatal and Postnatal Flavor Learning by Human Infants. Pediatrics, 107, 1-6. https://doi.org/10.1542/peds.107.6.e88

[13] Mennella, J.A., Griffin, C.E. and Beauchamp, G.K. (2004) Flavor Programming During Infancy. Pediatrics, 113, 840-845. https://doi.org/10.1542/peds.113.4.840

[14] Mennella, J.A., Lukasewycz, L.D., Castor, S.M. and Beauchamp, G.K. (2011) The Timing and Duration of a Sensitive Period in Human Flavor Learning: A Randomized Trial. American Journal of Clinical Nutrition, 93, 1019-1024. https://doi.org/10.3945/ajcn.110.003541

[15] Falciglia, G.A., Piazza, J.C., Ritcher, E., Reinerman, C. and Lee, S.Y. (2014) Nutrition Education for Postpartum Women: A Pilot Study. Journal of Primary Care and Community Health, 5, 275-278. https://doi.org/10.1177/2150131914528515

[16] Bandura, A. (1992) Social Cognitive Theory: An Agentic Perspective. Asian Journal of Social Psychology, 2, 21-41. https://doi.org/10.1111/1467-839X.00024

[17] US Department of Agriculture (2005) My Pyramid: Steps to a Healthier you. Center 
for Nutrition Policy and Promotion, CNPP-15.

[18] Butte, N., Cobb, K., Dwyer J., Graney, L., Heird, W. and Rickard, K. (2004) The Start Healthy Feeding Guidelines for Infants and Toddlers. Journal of the American Dietetic Association, 104, 442-454. https://doi.org/10.1016/j.jada.2004.01.027

[19] Millen, B. and Morgan, J.L. (1996) The 2D Food Portion Visual. Nutrition and Consulting Enterprises, Framingham, MA.

[20] Fox, M.K., Pac, S., Devaney, B. and Jankowski, L. (2004) Feeding Infants and Toddlers Study: What Foods are Infants and Toddlers Eating? Journal of the American Dietetic Association, 104, 22-30. https://doi.org/10.1016/j.jada.2003.10.026

[21] Ogden, C.L., Kuckzmarski, R.J. and Flegal, K.M. (2002) Centers for Disease Control and Prevention 2000 Growth Charts for the United States: Improvements to the 1977 National Center for Health Statistics Version. Pediatrics, 109, 45-60. https://doi.org/10.1542/peds.109.1.45

[22] Geraghty, S.R., Davidson, B.S., Warne, B.B., Sapsford, A.L., Ballard, J.L., List, B.A., Akers, R. and Morrow, A.L. (2005) The Development of a Research Human Milk Bank. Journal of Human Lactation, 21, 59-66.

https://doi.org/10.1177/0890334404273162

[23] Ammerman, A.S., Lindquist, C.H., Lohr, K.N. and Hersey, J. (2002) The Efficacy of Behavioral Interventions to Modify Dietary Fat and Fruit and Vegetable Intake: A Review of the Evidence. Preventive Medicine, 35, 25-41.

[24] Thomson, C.A. (2011) A Systematic Review of Behavioral Interventions to Promote Intake of Fruit and Vegetables. Journal of the American Dietetic Association, 111, 1523-1535. https://doi.org/10.1016/j.jada.2011.07.013 
Supplemental Table 1. Sensitivity analyses for the intervention effect on maternal total target vegetable consumption (primary outcome).

$\begin{array}{cccccc}\Delta_{\text {Intervention }}-\Delta_{\text {Usual }} & \Delta_{\text {Intervention }}-\Delta_{\text {Usual }} & \Delta_{\text {Intervention }}-\Delta_{\text {Usual }} & P \text {-value } & P \text {-value } & P \text {-value } \\ \text { Mean (SE) } & \text { Mean (SE) } & \text { Mean (SE) } & 6 \text { mo. } & 12 \text { mo. } & 18 \text { mo. } \\ 6 \text { mo. } & 12 \mathrm{mo} . & 18 \mathrm{mo} . & & & \end{array}$

\section{Maternal total target vegetables (servings/d)}

${ }^{a}$ Adjusted for baseline energy intake $(\mathrm{kcal} / \mathrm{d})$

$0.8(0.2)$

$0.6(0.2)$

$0.3(0.2)$

$<0.01$

$<0.01$

0.04

${ }^{\mathrm{b}}$ Multiple imputation model incorporating auxiliary variables predictive of withdrawal

$0.8(0.2)$

$0.4(0.2)$

$<0.01<0.01$

0.06

cPattern-mixture model

$0.5(0.2)$

$0.4(0.2)$

$0.3(0.1)$

$<0.01 \quad 0.02$

0.03

Abbreviations: SE, standard error; ANCOVA, analysis of covariance; Notes: All models represent intention-to-treat analysis under different assumptions for the missing data; ${ }^{a}$ Maximum likelihood-based estimates obtained from linear mixed-effects model including treatment, time, baseline response, baseline energy, and a time by treatment interaction term as fixed effects; ${ }^{\mathrm{b}}$ ANCOVA estimates including treatment and baseline response as covariates and modeling change from baseline as the dependent variable. Factors predictive of withdrawal including age, ever breastfeeding, education, marital status, and race were included in the multiple imputation model as auxiliary variables when imputing monotone missing values of target vegetables $(n=20$ datasets imputed); 'Pattern-mixture model utilizes the control distribution of total target vegetable intake to impute missing values for participants in the intervention arm who withdrew.

Submit or recommend next manuscript to SCIRP and we will provide best service for you:

Accepting pre-submission inquiries through Email, Facebook, LinkedIn, Twitter, etc. A wide selection of journals (inclusive of 9 subjects, more than 200 journals)

Providing 24-hour high-quality service

User-friendly online submission system

Fair and swift peer-review system

Efficient typesetting and proofreading procedure

Display of the result of downloads and visits, as well as the number of cited articles

Maximum dissemination of your research work

Submit your manuscript at: http://papersubmission.scirp.org/

Or contact ojog@scirp.org 